\title{
Effect of Nonionic Detergents on Lipoxygenase Catalysis
}

\author{
Maria J. Schilstra, Gerrit A. Veldink* and Johannes F.G. Vliegenthart \\ Bijvoet Center for Biomolecular Research, Department of Bio-Organic Chemistry, Utrecht University, The Netherlands
}

In many studies on lipoxygenase catalysis, nonionic detergents are used to obtain an optically transparent solution of the fatty acid substrate. In order to resolve some controversies that exist with regard to the interpretation of kinetic data obtained with solutions containing nonionic detergents, a systematic investigation was undertaken into the effects of Lubrol, Tween-20 and Triton X-100 $(0-0.8 \mathrm{~g} / \mathrm{L})$ on the kinetics of linoleate (2.5-110 $\mu \mathrm{M})$ dioxygenation, catalyzed by lipoxygenase-1 or lipoxygenase-2 from soybean, at $\mathrm{pH} 9$ or 10 , at $25^{\circ} \mathrm{C}$. Under most conditions, it was found that the detergents slowed down the reaction. However, at high linoleate concentrations, where substrate inhibition of lipoxygenase is significant, small amounts of detergent increased the dioxygenation rate. In a quantitative analysis of the results, a kinetic model in which the incorporation of linoleate in the detergent micelles is formulated as a simple reversible equilibrium, and in which both lipoxygenase-1 and -2 interact with free linoleate, but not with linoleate incorporated in the micelles, appeared to be sufficient to predict experimental results over a wide range of experimental conditions. According to this model, the changes in the dioxygenation kinetics caused by the presence of nonionic detergents are similar (but not equal) to those caused by competitive inhibitors. The conclusions that monomeric, nonmicellar linoleate is the preferred substrate for lipoxygenase and that the observed inhibition and stimulation are solely due to changes in the effective linoleate concentration strongly corroborate the earlier observations by Galpin and Allen [Biochim. Biophys. Acta 488 (1977), 392-401].

Lipids 29, 225-231 (1994).

Lipoxygenases (EC 1.13.11.12) catalyze the dioxygenation of polyunsaturated fatty acids that contain one or more $(1 Z, 4 Z)$-pentadiene systems. The reaction products are hydroperoxides with a conjugated $(E, Z)$-diene moiety (for reviews see Refs. 1-3). Soybean lipoxygenase-1, the most thoroughly characterized of all plant lipoxygenases, shows optimum activity at $\mathrm{pH} 9-11$. At these $\mathrm{pH}$ values, the critical micelle concentration (CMC) of

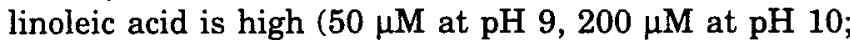
Ref. 4). However, many other lipoxygenases have a $\mathrm{pH}$ optimum around 7. At pH 7, the CMC of linoleic acid is much lower $(<20 \mu \mathrm{M}$, Ref. 4$)$, and the solutions are often

\footnotetext{
*To whom correspondence should be addressed at the Bijvoet Center for Biomolecular Research, Department of Bio-Organic Chemistry, Utrecht University, P.O. Box 80.075, NL-3508 TP Utrecht, The Netherlands.

Abbreviations: $\mathrm{CMC}$, critical micelle concentration; $\mathrm{CMC}_{\mathbf{d}}$, concentration of nonmicellar detergent; HPOD, hydroperoxyoctadecadienoic acid; SDS, sodium dodecylsulfate.
}

turbid. In studies on lipoxygenases at neutral $\mathrm{pH}$, nonionic detergents, such as Tween-20 or Triton X-100 (source information in Experimental Procedures section), are frequently added to the reaction mixture to obtain an optically transparent solution.

Detergents are known to affect the dioxygenation rate. Many investigators have observed inhibition and/or stimulation of the dioxygenation reaction by surfactants (e.g., Refs. 5-8), but controversy exists about the interpretation of the data. In two recent studies on the effect of surfactants on the lipoxygenase reaction $(7,8)$, the observed inhibition and activation were attributed to direct interactions between the detergents and lipoxygenase. In their analysis of the data, the authors did not take into account the fact that linoleate is itself an amphiphile and can, therefore, interact with the detergent micelles. On the other hand, in 1977 Galpin and Allen (9) published a study on the influence of micelle formation on lipoxygenase kinetics in which they used $n$-alcohols and $n$-carboxylic acids to modify the CMC of linoleate. Their results provided convincing evidence that lipoxygenase interacts mainly with monomeric linoleate, and that virtually all of the inhibition or activation effects of the alcohols and carboxylic acids could be attributed to changes in the concentration of nonmicellar linoleate. Furthermore, in a recent analysis of lipoxygenase catalysis in reversed micelles, Perez-Gilabert et al. (10) found that their data were best predicted by a model in which linoleate dissolved in the water phase was the sole substrate for lipoxygenase-1, and interactions between lipoxygenase and interfacial linoleate were neglected.

In order to resolve the controversies concerning the interpretation of dioxygenation data collected in the presence of a detergent, we have studied the dioxygenation kinetics over a wide range of linoleic acid and detergent concentrations. In most experiments we have used the nonionic detergent Lubrol (source information in Experimental Procedures section), which does not absorb at the wavelength used to monitor the dioxygenation $(243 \mathrm{~nm})$, but we have also studied the effect of two other commonly used detergents, Tween-20 and Triton $\mathrm{X}-100$. Furthermore, we recorded the $\mathrm{pH}$ profile for lipoxygenase-1 activity in buffers containing detergent and compared it with the profile obtained in the absence of detergent.

We present a quantitative analysis of the data obtained at $\mathrm{pH} 9$ and 10. Because the Michaelis-Menten formulation, which is often used to describe dioxygenation data, is only valid under certain conditions (11), we have analyzed the data in terms of the more comprehensive two-step model for lipoxygenase catalysis (11-13). 


\section{EXPERIMENTAL PROCEDURES}

Materials. Lipoxygenase-1 was prepared from soybeans (White Hilum; Central Soya, Utrecht, The Netherlands) as described previously (Ref. 11 and references therein). The preparation had a specific activity of $200 \mu \mathrm{mol}$. $\mathrm{min}^{-1} \cdot \mathrm{mg}^{-1}$ at $\mathrm{pH} 9$, and of $40 \mu \mathrm{mol} \cdot \mathrm{min}^{-1} \cdot \mathrm{mg}^{-1}$ at $\mathrm{pH}$ 7 (measured at $100 \mathrm{mM}$ linoleate). It showed one major band after sodium dodecylsulfate (SDS) gel electrophoresis upon Coomassie Brilliant Blue staining. Lipoxygenase-1 was stored in $0.1 \mathrm{M}$ sodium acetate, $\mathrm{pH}$ 5.5 , containing $134 \mathrm{~g} / \mathrm{L}$ ammonium sulfate. Partially purified lipoxygenase-2 was obtained in the second ion exchange chromatographic step (DEAE-Sepharose; Pharmacia, Uppsala, Sweden) in the same purification procedure. Lipoxygenase-2 activity was found in a small peak preceding the lipoxygenase-1 peak. The protein in the lipoxygenase-2 peak was precipitated with $320 \mathrm{~g} / \mathrm{L}$ ammonium sulfate, then resuspended in $0.1 \mathrm{M}$

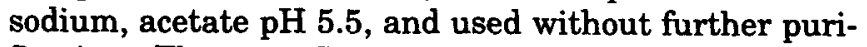
fication. The specific activity of the lipoxygenase-2 preparation was $1 \mathrm{mmol} \cdot \mathrm{min}^{-1} \cdot \mathrm{mg}^{-1}$ at $\mathrm{pH} 6$ (measured at the linoleic acid concentration at which the rate is maximal, $25 \mathrm{mM}$, see Fig. 3a later in article) and was $0.3 \mathrm{mmol} \cdot \mathrm{min}^{-1} \cdot \mathrm{mg}^{-1}$ at $\mathrm{pH} 9$.

Linoleic acid $[(9 Z, 12 Z)$-octadecadienoic acid] was obtained from Janssen Chimica (Beerse, Belgium) and was stored under argon as a $300 \mathrm{mM}$ solution in methanol p.a. (Merck, Darmstadt, Germany) at $4^{\circ} \mathrm{C}$. Lubrol (unspecified polyoxyethyleneglycols from fatty alcohols) was purchased from ICN (Cleveland, Ohio), Tween-20 from Fluka (Buchs, Switzerland), and Triton X-100 from Serva (Heidelberg, Germany).

Kinetic measurements. A Hi-Tech (Salisbury, United Kingdom) SF-51 stopped-flow spectrophotometer was used for the kinetic experiments. The formation of hydroperoxylinoleate (HPOD) was followed by recording the absorbance at $243 \mathrm{~nm}$ (UG-5 filter, $45 \%$ transmission at $243 \mathrm{~nm}$ ) in a 10 - $\mathrm{mm}$ light-path observation chamber. The molar absorbance for HPOD at $243 \mathrm{~nm}$ was $17.8 \times 10^{3} \mathrm{M}^{-1} \cdot \mathrm{cm}^{-1}$. The absorbances at $243 \mathrm{~nm}$ of $1 \mathrm{~g} / \mathrm{L}$ Tween-20 and $1 \mathrm{~g} / \mathrm{L}$ Triton X-100 were 0.15 and $0.48 \mathrm{~cm}^{-1}$, respectively. Measurements were performed at $25^{\circ} \mathrm{C}$ in $0.09 \mathrm{M}$ Na-borate $(\mathrm{pH} 7.9-10.7)$ or $0.09 \mathrm{M}$ sodium phosphate (pH 6.7-9.0) buffers. A small amount of methanol from the linoleic acid stock solution was carried through into the final reaction mixture (maximally $0.03 \% \mathrm{vol} / \mathrm{vol}$ ). As methanol is known to have a slight effect on the kinetics of the lipoxygenase reaction (11), the methanol concentration was kept constant $(0.03 \% \mathrm{vol} / \mathrm{vol})$ in all reaction mixtures by adding an appropriate amount of extra methanol. The reactions were initiated by mixing lipoxygenase (in syringe 1) with linoleate and detergent (in syringe 2). The final lipoxygenase concentration was $50 \mathrm{nM}$, unless otherwise indicated.

Interpretation of the data. An analytical steady-state rate equation for lipoxygenase catalyzed oxygenation of linoleate was derived in previous studies $(11,13)$. The steady-state rate, $r$, at given linoleate $(S)$ and HPOD $(P)$ concentrations is given by:
$r=\frac{V_{\max }[S]}{\left.\left\{K_{S}^{*}\left(1+[P] / K_{P}^{*}\right)+[S]\right\}+\alpha \cdot\left\{K_{P}\left(1+\left[S y / K_{S}\right)+[P]\right\} \cdot[S\rangle\right\} P\right]}[1]$

In this expression, $V_{\max }$ is the maximum rate, $K_{S}^{*}, K_{P}^{*}, K_{S}$ and $K_{P}$ are the dissociation constants of the iron(III)lipoxygenase- $\left(K^{*}\right)$ and iron(II)-lipoxygenase $(K)-S$ and $-P$ complexes. The parameter $\alpha$ is a constant, provided $\left[\mathrm{O}_{2}\right]$ does not change by more than $20 \%$.

All observed curves showed an initial increase in rate (the induction period), followed by a short period of maximum rate, $r_{\max }$ (note that $r_{\max }$ is not equal to $V_{\max }$ ). Numerical simulations of the lipoxygenase reaction indicate that the steady-state approximation may be applied in analyzing $r_{\max }$ as a function of [linoleate] and [HPOD] (13). The observed values of $r_{\max }$ (indicated as $r$ in the rest of the text) were fitted to the steady-state rate equation using a nonlinear least-squares program (Levenberg-Marquardt algorithm, Ref. 14) for evaluating functions of multiple independent variables. The values for $[S]$ and $[P]$ used in the fitting procedure were estimated from the absorbance at $243 \mathrm{~nm}$ at the start of the maximum rate period. Since the concentration of $\mathrm{O}_{2}$ in water, at $25^{\circ} \mathrm{C}$ under air, is $240 \mu \mathrm{M}$, and $r$ is measured at $3 \mu \mathrm{M}<[P]<5 \mu \mathrm{M},\left[\mathrm{O}_{2}\right]$ varies less than $5 \%$ in the experiments, and therefore the value of $\alpha$ is constant within the experimental error.

\section{RESULTS}

Effect of Lubrol on the dioxygenation rate at alkaline $p H$. The dioxygenation of 2.5-110 $\mu \mathrm{M}$ linoleate, catalyzed by $50 \mathrm{nM}$ soybean lipoxygenase-1 at $\mathrm{pH} 10$, was recorded in the absence of detergent. After completion of the reaction, all linoleate (within the experimental error of $5 \%$ ) appeared to be converted into HPOD. The steady-state rates are shown in Figure 1a. The values of $V_{\max }$ and $K_{S}{ }^{*}$ were estimated from a nonlinear least-squares fit of these data to Equation 1 (Experimental Procedures section). Because the other parameters are highly correlated under the conditions of the experiments $\left(\left[\mathrm{O}_{2}\right]=\right.$ $240 \mu \mathrm{M}$ and $[\mathrm{HPOD}]<5 \mu \mathrm{M}$ ), they were entered as constants: $K_{P}^{*}=25 \mu \mathrm{M}(11,13), K_{P}=15 \mu \mathrm{M}(15), K_{S}=20 \mu \mathrm{M}$ and $\alpha=0.01(11,13)$. The best fit values for $V_{\max }$ and $K_{S}^{*}$ were $15.0 \pm 0.2 \mu \mathrm{M} / \mathrm{s}$ and $20 \pm 1 \mu \mathrm{M}$, respectively (Chi square $=1.5$ ).

In Figure 1b we illustrate the effect of increasing concentrations of Lubrol $(0.025-0.8 \mathrm{~g} / \mathrm{L})$ on the steady-state rate (r) at four different linoleate concentrations $(9,30$, 55 and $110 \mu \mathrm{M})$. At all linoleate concentrations, the presence of detergent decreases the dioxygenation rate. However, all of the linoleate is eventually converted into HPOD. The effect of Lubrol at a concentration of $1.7 \mathrm{nM}$ lipoxygenase is the same as the effect at $50 \mathrm{nM}$. The $r_{\max }$ values measured at $50 \mathrm{nM}$ lipoxygenase were 30 times larger than the values measured at $1.7 \mathrm{nM}$ lipoxygenase at all Lubrol concentrations.

The data presented in Figure 1 are not compatible with two simple inhibition modes in which the detergent acts directly on the enzyme (uncompetitive and simple noncompetitive inhibition). Competitive inhibition or a more 

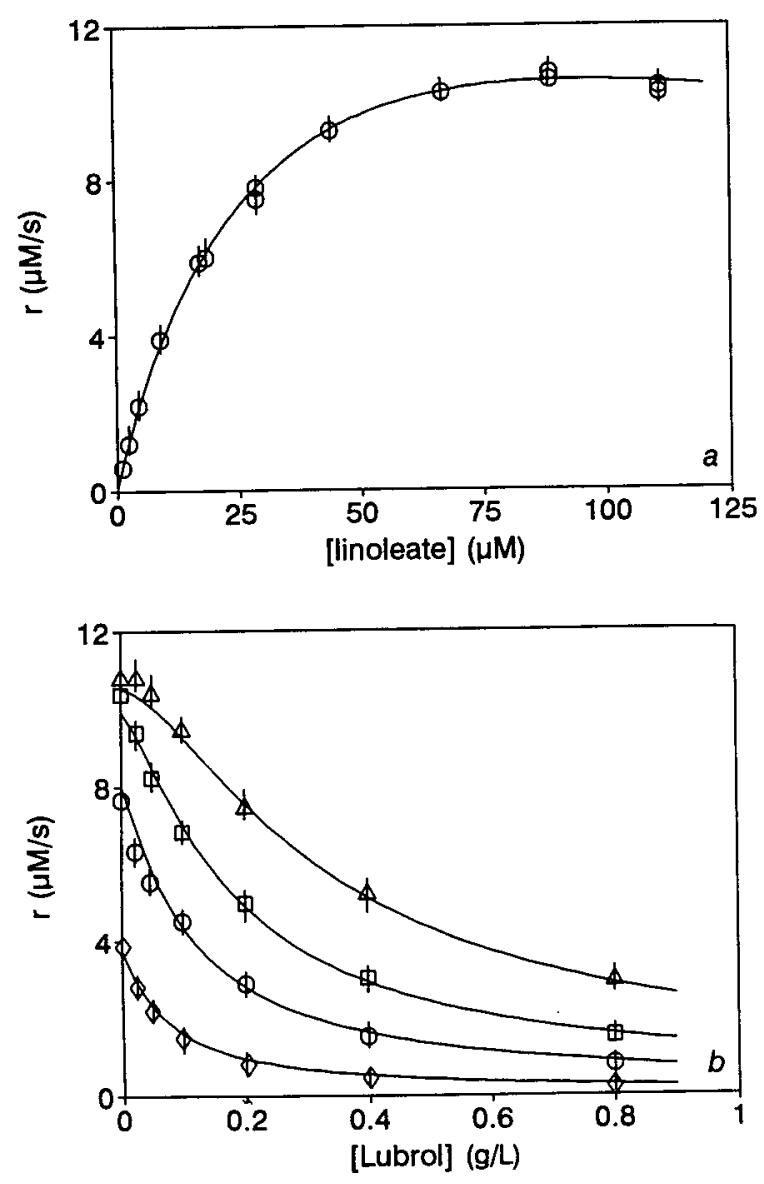

FIG. 1. Effect of Lubrol on the dioxygenation of linoleate catalyzed by $50 \mathrm{nM}$ lipoxygenase-1. Reactions were carried out in $0.1 \mathrm{M}$ sodium borate at $\mathrm{pH} 10$. $a$ : Steady-state rates in the absence of Lubrol at varying linoleate concentrations; circles, observed rates; solid lines show rates calculated with $V_{\text {max }}=15 \mu \mathrm{M} / \mathrm{s}, K_{S}^{*}=20, K_{P}^{*}=25, K_{S}=20, K_{P}=15 \mu \mathrm{M}$ and $\alpha=0.01$. $b$ : Steady-state rates measured at different Lubrol concentrations, starting with 9 (diamonds), 30 (circles), 55 (squares) and 110 (triangles) $\mu \mathrm{M}$ linoleate. Solid lines: rates calculated with the parameters mentioned under $(a)$ and $K_{D}=32 \mu \mathrm{M}, f=770 \mu \mathrm{mol} / \mathrm{g}$ and $\mathrm{CMC}_{\mathrm{d}}=0 . \mathrm{CMC}_{\mathrm{d}}$, concentration of nonmicellar detergent.

complicated mode of enzyme inactivation is not excluded by the data. However, the presence of detergent would also slow down the reaction if micelle-bound linoleate were a poor substrate for lipoxygenase. Such a model would be in good agreement with the results of Galpin and Allen (9) and those of Perez-Gilabert et al. (10). In order to investigate this possibility, we used a kinetic model with the following features: (i) The fatty acid molecules interact with "fatty acid binding sites" on the detergent micelles in a simple reversible equilibrium, described by the dissociation constant $K_{D}$, (ii) only micelles can bind the fatty acid molecules, monomeric detergent molecules have no effect; (iii) the micelle-bound form of the fatty acid cannot interact with the enzyme; (iv) reactions between the fatty acid molecules and the detergent micelles are much faster than the enzyme-catalyzed reaction, and are not, therefore, rate limiting. For the sake of simplicity, we assume that the concentration of nonmicellar detergent $\left(\mathrm{CMC}_{\mathrm{d}}\right)$ is constant at all linoleate concentrations. If $1 \mathrm{~g}$ of detergent can adsorb $f \mu$ moles of fatty acid, then $B_{0}$, the total concentration of "binding sites," is equal to $f \cdot\left(D-C C_{d}\right)$, where $D$ is the concentration of detergent in $\mathrm{g} / \mathrm{L}$. At a total fatty acid concentration $A_{0} \mu \mathrm{M}$, the concentration of free fatty acid $[A]$ is given by:

$$
[A]=\left\{\left[\left(K_{D}+B_{0}-A_{0}\right)^{2}+4 \cdot K_{D} \cdot A_{0}\right]^{1 / 2}-\left(K_{D}+B_{0}-A_{0}\right)\right\} / 2
$$

In the nonlinear least-squares fit of the data with this model, the concentrations of free linoleate and HPOD ([S] and $[P])$ for use in Equation 1 were calculated from Equation 2 by substituting the appropriate total concentrations $S_{0}$ and $P_{0}$ for $A_{0}$. All data presented in Figure 1 were evaluated simultaneously (see Experimental Procedures section). The accuracy of the data does not allow separate estimations of $K_{D}$ for Lubrol-linoleate and Lubrol-HPOD, because under the conditions of the measurements $(3<$ [HPOD] $5 \mu \mathrm{M})$, the rate is mainly determined by the concentration of linoleate. The best fit values are: $V_{\max }=15.0 \pm 0.2 \mu \mathrm{M} / \mathrm{s}, K_{S}^{*}=20 \pm 1 \mu \mathrm{M}, f=$ $770 \pm 80 \mu$ moles $/ g$ and $K_{D}=32 \pm 5 \mu \mathrm{M}$ (Chi square $=2$, but see below). The concentration of noninteracting detergent $\left(\mathrm{CMC}_{\mathrm{d}}\right)$ must be very small, and cannot be determined accurately from these data. Its upper limit is estimated to be $10 \mathrm{mg} / \mathrm{L}$. The experimental data satisfy the simple model very well. This can be seen in Fig ure $1 \mathrm{~b}$, in which the calculated data have also been indicated.

The values of $K_{D}$ and $f$ appeared to be correlatedother values of $f$ and $K_{D}$ also fit the data well, as long as $f / K_{D}$ is approximately $25 \mathrm{~L} / \mathrm{g}$ and $K_{\mathrm{D}}>15 \mu \mathrm{M}$. Therefore, the absolute values of $K_{D}$ and $f$ (and their errors) have little physical significance. The correlation indicates that the total number of binding sites $B_{0}$ is much larger than the number of occupied sites, $[A D]$, even at the highest concentration of linoleate used in these experiments. When $B_{0}-[A D] \approx B_{0}$, the expression for the concentration of free linoleate becomes:

$$
[A]=A_{0}\left(1+\left(D-\mathrm{CMC}_{\mathrm{d}}\right) \cdot f / K_{D}\right)
$$

When Equation 3 was used instead of Equation 2 for the calculation of free linoleate, the fit of the data was equally good (Chi square $=2$ ). The best fit value for $f / K_{D}$ was $21 \pm 5 \mathrm{~L} / \mathrm{g}$.

When the rate is measured as a function of the linoleate concentration and the concentration of detergent is constant, the observed rates coincide with the predicted values within the experimental error. However, at low substrate concentrations $(<15 \mu \mathrm{M})$, the observed rates are systematically somewhat lower, and at high substrate concentrations $(50-100 \mu \mathrm{M})$, the values are slightly higher than the predicted rates (not shown). This effect may be due to exclusion phenomena such as those described, for example, by McGhee and Von Hippel (16). Binding of one molecule of linoleate to a detergent micelle presumably excludes binding of other linoleate molecules to potential "binding sites" in its vicinity. In that case, $f / K_{D}$ is a function of the linoleate 


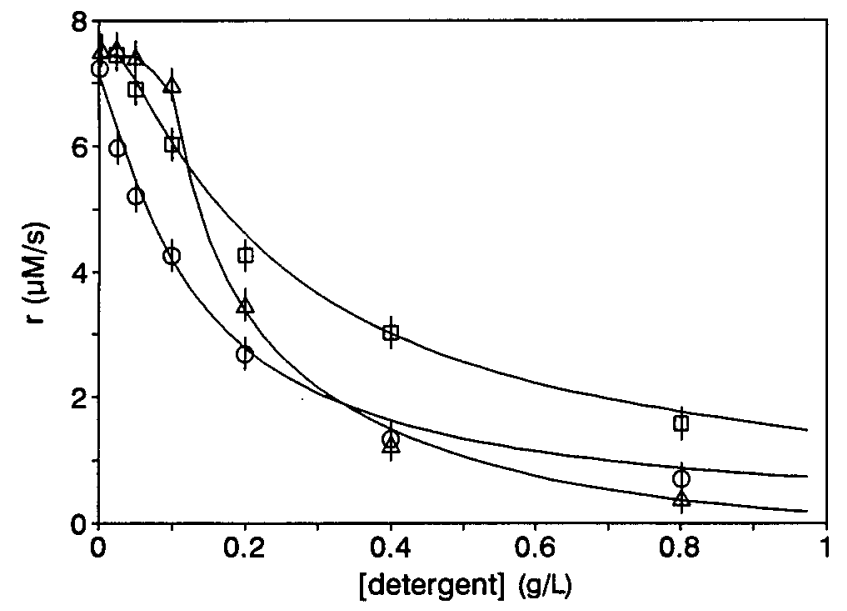

FIG. 2. Comparison of the effects of Lubrol (circles), Tween20 (squares) and Triton X-100 (triangles) on the steady-state oxygenation rates. Reactions were performed with $50 \mathrm{nM}$ lipoxygenase-1 and $30 \mu \mathrm{M}$ linoleate in $0.1 \mathrm{M}$ sodium borate, pH 10. Solid lines show rates calculated with parameters obtained from best fits of data obtained at 10,30,50 and 100 $\mu \mathrm{M}$ linoleate: $f / K_{D}=24 \mathrm{~L} / \mathrm{g}, \mathrm{CMC}_{\mathrm{d}}=0$ (for Lubrol data); f/K $=10 \mathrm{~L} / \mathrm{g}, \mathrm{CMC}_{\mathrm{d}}=0.031 \mathrm{~g} / \mathrm{L}$ (for Tween-20 data) and $f / K_{D}=30$ $\mathrm{L} / \mathrm{g}$ and $\mathrm{CMC}_{\mathrm{d}}=0.091 \mathrm{~g} / \mathrm{L}$ (for Triton X-100 data). All other parameters and abbreviation are as in Figure 1.

concentration rather than a constant, and the estimated value for $f / K_{D}$ is merely an average.

Effect of other nonionic detergents. Because Triton X-100 and Tween-20 are also commonly used to solubilize linoleic acid, we investigated whether the model described for the action of Lubrol holds for these compounds. We measured the dioxygenation rates at 10,30 , 50 and $100 \mu \mathrm{M}$ linoleate, in the presence of $0-0.8 \mathrm{~g} / \mathrm{L} \mathrm{de}-$ tergent. In Figure 2 the effects of increasing concentrations of Tween-20 or Triton X-100 are compared with the results obtained for Lubrol (see Fig. 1b, linoleate concentration, $30 \mu \mathrm{M}$ ). Addition of less than approximately $0.05 \mathrm{~g} / \mathrm{L}$ Tween-20 or $0.1 \mathrm{~g} / \mathrm{L}$ Triton X-100 has little effect (all linoleate concentrations), but higher concentrations of either detergent cause the dioxygenation rate to decrease. After completion of the reactions, all of the linoleate (within the experimental error of approximately $5 \%$ ) had again been converted into HPOD. The data for Tween-20 and for Triton X-100 (all linoleate concentrations) were successfully fitted to Equations 1 and 2. The best fit values for $K_{D}$ and $f$ were correlated, and the use of Equation 3 instead of Equation 2 gave equally good results. The best fit values of $f / K_{D}$ are $10 \mathrm{~L} / \mathrm{g}$ for the Tween-20 data, and $30 \mathrm{~L} / \mathrm{g}$ for the Triton $\mathrm{X}-100$ data. The best fit values for $\mathrm{CMC}_{\mathrm{d}}$ were $0.031 \pm$ 0.008 and $0.091 \pm 0.003 \mathrm{~g} / \mathrm{L}$, respectively. These values are lower than the CMCs that have been reported for these detergents in pure form $(0.06 \mathrm{~g} / \mathrm{L}$ for Tween-20 and $0.19 \mathrm{~g} / \mathrm{L}$ for Triton X-100, Refs. 17 and 18). However, the concentration of monomeric detergent in equilibrium with mixed micelles is always lower than the CMC of the pure detergent (19). Furthermore, we used commercial detergent preparations, whose CMCs may have been smaller than the published values, owing to inhomogeneity or impurities.
Effect of Lubrol on the lipoxygenase-2 reaction. If the inhibition of the dioxygenation reaction by Lubrol is solely due to adsorption of the fatty acid substrate, then the effect of Lubrol on the reaction catalyzed by other lipoxygenases must be predictable from the data obtained with lipoxygenase-1. Therefore, we investigated
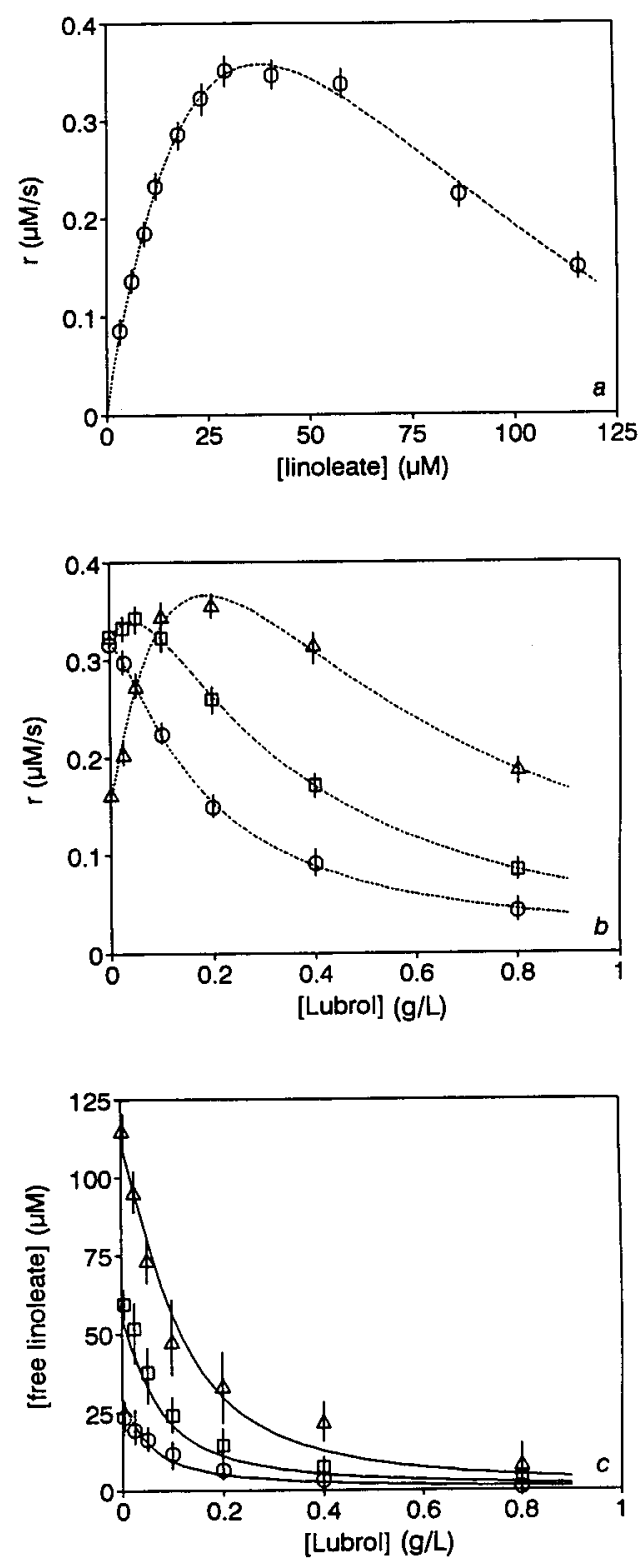

FIG. 3. Effect of Lubrol on the dioxygenation of linoleate, catalyzed by lipoxygenase-2. Data obtained with a partially purified enzyme preparation in $0.1 \mathrm{M}$ sodium borate at $\mathrm{pH}$ 9. $a$ : Rates in the absence of Lubrol at varying linoleate concentrations. $b$ : Rates measured in the presence of varying Lubrol concentrations, starting with 30 (circles), 55 (squares) and 110 (triangles) $\mu \mathrm{M}$ linoleate. c: Comparison of concentrations of free (nonmicellar) linoleate, estimated from the data obtained with lipoxygenase-2 with calculated values. Solid lines: calculated concentrations $\left(K_{D}=32 \mu \mathrm{M}\right.$ and $f=770 \mu \mathrm{mol} / \mathrm{g}$ ). Other symbols: concentration of free linoleate estimated by comparing the rates from $b$, those obtained in the absence of Lubrol $(a)$. Total linoleate concentrations: 30 (circles), $\mathbf{5 5}$ (squares) and 110 (triangles) $\mu \mathrm{M}$. 
the effect of Lubrol on the reaction catalyzed by lipoxygenase-2 from soybean.

Lipoxygenase-1 and -2 have different substrate specificities (20) and form different products (21). In the lipoxygenase-2 reaction, $10-35 \%$ of all linoleate is converted into compounds other than HPOD (see Ref. 22), as judged by the increase of absorbance at $285 \mathrm{~nm}$ during the reaction (in the lipoxygenase- 1 reaction, over 95\% of the linoleate is converted into HPOD). The steady-state kinetics are also different. A plot of the steady-state dioxygenation rate for lipoxygenase-2 as a function of linoleate concentration, obtained at $\mathrm{pH} 9$ and at a protein concentration of $0.25 \mu \mathrm{g} / \mathrm{mL}$, is shown in Figure 3a. The curve shows a marked decrease in rate at linoleate concentrations greater than $25 \mu \mathrm{M}$. A similar decrease in rate appears in the lipoxygenase- 1 reaction, but only at linoleate concentrations above $100 \mu \mathrm{M}$ (not shown). The rate decrease is due to substrate inhibition (11). This effect is much more pronounced for lipoxygenase-2 reaction than for lipoxygenase-1.

Addition of Lubrol to the lipoxygenase-2 reaction mixture merely slows down the reaction when the linoleate concentration is $30 \mu \mathrm{M}$ (Fig. 3b). However, at a linoleate concentration of $55 \mu \mathrm{M}$, a slight stimulation of the reaction is observed at Lubrol concentrations up to $0.05 \mathrm{~g} / \mathrm{L}$. Concentrations greater than $0.1 \mathrm{~g} / \mathrm{L}$ are again inhibitory. A much larger stimulation is observed in the reaction with $110 \mu \mathrm{M}$ linoleate. At a Lubrol concentration of $0.2 \mathrm{~g} / \mathrm{L}$, the rate is twice as high as in the absence of detergent. At higher Lubrol concentrations, the reaction proceeds more slowly than at $0.2 \mathrm{~g} / \mathrm{L}$ Lubrol. In spite of the apparently different effects of Lubrol on lipoxygenase- 1 and -2 , the results can still be explained with the same model. The micelles adsorb a certain fraction of the linoleate, which cannot then be bound by lipoxygenase. When the total linoleate concentration is smaller than $25 \mu \mathrm{M}$, addition of Lubrol only results in a reduced reaction rate. At higher linoleate concentrations, substrate inhibition of lipoxygenase-2 is substantial. Decreasing the concentration of free linoleate will result in a decreased substrate inhibition and, thus, in a rate increase. Addition of more detergent eventually results in a rate decrease, because the concentration of free linoleate drops below $25 \mu \mathrm{M}$.

Because of the lack of sufficient a priori knowledge of the values of $K_{P}^{*}, K_{S}, K_{P}$ and $\alpha$ for the lipoxygenase-2 reaction, it is not possible to perform a quantitative analysis similar to the one applied to the lipoxygenase-1 data. Assuming that the model for the action of detergents is correct, it is possible to estimate the concentrations of free linoleate simply by comparing the rates observed in the presence of Lubrol with the values obtained in the absence of detergent (Fig. 3a). The dioxygenation rate in the absence of detergent at $35-60 \mu \mathrm{M}$ linoleate is approximately constant $(0.35 \pm 0.2 \mu \mathrm{M} / \mathrm{s})$. Therefore, the estimate of the concentration of free linoleate corresponding to a rate of $0.35 \pm 0.2 \mu \mathrm{M} / \mathrm{s}$ is rather inaccurate $(48 \pm 13 \mu \mathrm{M})$. The error on the other estimates is smaller (approximately $5 \mu \mathrm{M}$ for estimates smaller than $25 \mu \mathrm{M}$, $10 \mu \mathrm{M}$ for estimates above $70 \mu \mathrm{M})$. In Figure $3 \mathrm{c}$ we compare these estimates with values calculated from Equa-
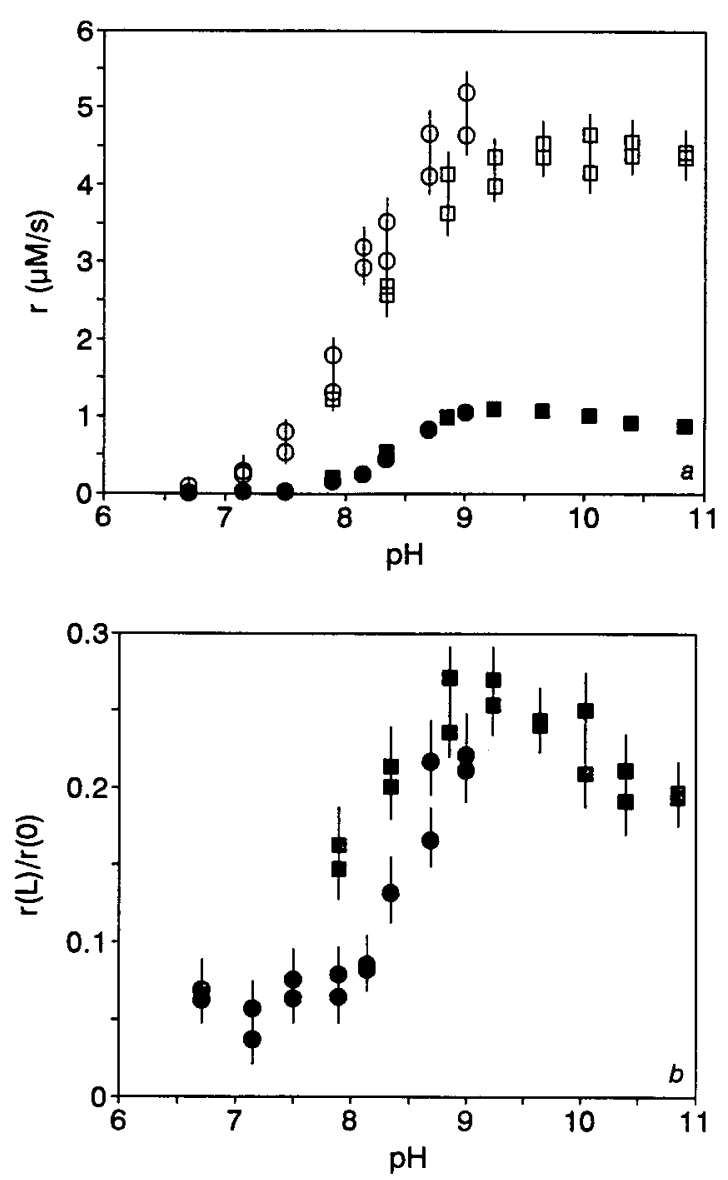

FIG. 4. Effect of pH. a: Steady-state dioxygenation rates in the absence or presence of $0.2 \mathrm{~g} / \mathrm{L}$ Lubrol. The concentration of lipoxygenase-1 was $50 \mathrm{nM}$, the total linoleate concentration was $10 \mu \mathrm{M}$. Open symbols, no Lubrol; closed sym. bols, $0.2 \mathrm{~g} / \mathrm{L}$ Lubrol; circles, $0.1 \mathrm{M}$ Na-phosphate buffers; squares, $0.1 \mathrm{M}$ Na-borate buffers. $b$ : Ratio $\mathbf{r}(\mathrm{L}) / \mathbf{r}(0)$ of rates in buffers with $[r(L)]$ and without $[r(0)] 0.2 \mathrm{~g} / \mathrm{L}$ Lubrol.

tion 2 , with $K_{D}=32 \mu \mathrm{M}$ and $f=770 \mu \mathrm{mol} / \mathrm{g}$, the parameter values calculated for the lipoxygenase-1 reaction. The concentrations of free linoleate estimated from the dioxygenation rates agree very well with the ones calculated with Equation 2. This indicates that the fraction of linoleate incorporated in micelles is independent of the type of lipoxygenase used in the assay.

The effect of $p H$. The effect of detergent on the $\mathrm{pH}$ profiles of lipoxygenase-1 is shown in Figure 4. The profile was obtained at $10 \mu \mathrm{M}$ linoleic acid. We used this lowlinoleate concentration to minimize effects due to substrate inhibition or aggregation. The plots show a steep increase in rate between $\mathrm{pH} 7$ and 9 . The rates measured in the presence of Lubrol are significantly lower (Fig. 4a). If the composition of the detergent-fatty acid micelles were independent of $\mathrm{pH}$, the concentration of free fatty acid would be the same at each $\mathrm{pH}$. In that case, the ratio of the rates in buffers with or without Lubrol would be constant. However, this ratio varies quite strongly (Fig. 4b). At $\mathrm{pH}<7.5$, dioxygenation in the absence of Lubrol is approximately 20 times faster than in the presence of Lubrol. At $\mathrm{pH}>9$, the rate in the absence 
of Lubrol is only 4 to 5 times higher. This means that the concentration of free lipid substrate is significantly smaller at $\mathrm{pH}<7.5$ than at $\mathrm{pH}>9$.

It has been proposed (6) that the activity of soybean lipoxygenase-1 depends on the charge of the fatty acid substrate. Lipoxygenase-1 reacts best with charged substrates (such as the linoleate anion), but seems to have a much lower affinity for uncharged compounds (such as unionized linoleic acid). The apparent $\mathrm{p} K_{a}$ value for linoleic acid is $7.9(6)$. The $\mathrm{pH}$ profile of lipoxygenase-1 obtained in the absence of detergent is consistent with this. One would also expect that the amount of linoleic acid incorporated in the mixed micelles would decrease upon increasing the $\mathrm{pH}$, owing to the repulsive interactions between the negatively charged head groups. The curve of the ratios (Fig. 4b) has the same sigmoidal shape as the activity profile (Fig. 4a), because the presence of detergent merely exaggerates the effect of $\mathrm{pH}$ on the lipoxygenase-1 activity. However, the presence of Lubrol can cause a shift in the $\mathrm{pH}$ optimum of lipoxygenases that have highest affinity for less polar substrates (unpublished observations). The results presented here do not exclude the possibility that Lubrol has some $\mathrm{pH}$ dependent effect on lipoxygenase itself, nor that the $\mathrm{p} K_{a}$ of linoleic acid is affected by the presence of Lubrol. Nonetheless, the experiments indicate that $\mathrm{pH}$ profiles obtained in buffers containing detergent are different from those obtained in buffers without detergent.

\section{DISCUSSION}

The results presented above strongly favor a model in which lipoxygenase has a substantially higher affinity for monomeric substrate than for fatty acid incorporated in micelles. A quantitative model, in which the detergent simply decreases the effective substrate concentration, accurately predicts the results for more than one type of lipoxygenase, without requiring any further assumptions. It is not possible to reconcile the present results with an equally simple model in which a detergent acts directly on the enzyme. Such a model would require many assumptions, for which no experimental evidence exists, to explain the observed inhibition and stimulation.

The influence of micelle formation on lipoxygenase kinetics was studied by Galpin and Allen (9). Our findings confirm their conclusion that the effects of amphiphiles on the dioxygenation reaction could be ascribed to physicochemical interaction of these compounds with the substrate rather than to a direct interaction with the enzyme itself.

In many studies on lipoxygenase, a nonionic detergent is routinely added to solubilize the substrate. If quantitative conclusions are to be drawn from the experiments, it is essential to characterize the system in detail. When such a characterization is omitted, it is quite possible that phenomena caused by changes in the effective substrate concentration are incorrectly attributed to direct interactions between the enzyme and the detergent. For instance, the rate increase that is ob- served after addition of small amounts of detergent at high fatty acid concentrations (see Fig. 3) is easily mistaken for direct stimulation of the lipoxygenase activity (cf. Ref. 8). Furthermore, changes in $K_{m}$ and $V_{\max }$ may be erroneously interpreted as indicative for competitive inhibition, for the following reason: The steady-state rate equation (Equation 1) for lipoxygenase-1 catalysis simplifies to the Michaelis-Menten equation for linoleate concentrations below $75 \mu \mathrm{M}$ and HPOD concentrations from 3 to $5 \mu \mathrm{M}$, because the second term in the denominator and the value of $[P] / K_{P}^{*}$ are both small (11). Substitution of the substrate concentration in the Michaelis-Menten equation with the expression for $[A]$ from Equation 3 yields an equation that is identical to the expression for the rate in the presence of a competitive inhibitor. Therefore, the model predicts that, under certain conditions, the presence of detergents causes changes in $K_{m}$ and $V_{\max }$ that are very similar to those caused by competitive inhibitors. As the MichaelisMenten equation is only an approximation, straight lines generated in a Lineweaver-Burk plot will intersect slightly off the ordinate (which is generally interpreted as "mixed inhibition"; cf. Ref. 8). However, the results obtained with lipoxygenase-2 exclude competitive inhibition. A competitive inhibitor would enhance the effect of product and substrate inhibition, and merely decrease the dioxygenation rate.

In the interpretation of results of experiments in which detergents are used, it should also be taken into account that: (i) $\mathrm{pH}$ profiles obtained in buffers containing detergent are different from those obtained in buffers without detergent; (ii) lipoxygenase inhibitors and activators may associate with micelles and therefore seem to be less effective than in the absence of detergent; and (iii) it is possible that the addition of negatively charged amphiphiles to the nonionic detergent/linoleate solution will limit the amount of fatty acid that can be incorporated into the micelles, and thus stimulate the reaction (23). Positively charged amphiphiles may have the opposite effect (8).

It has been reported that some mammalian lipoxygenases are able to oxygenate biological membranes without prior action of a phospholipase (24). From the present study, it seems highly likely that lipoxygenase-1 and -2 from soybean interact preferentially with free linoleate, and not with linoleate included in a matrix of (nonionic) amphiphiles. Therefore, investigations are underway into the ability of plant lipoxygenases to oxygenate artificial and biological lipid bilayers.

\section{ACKNOWLEDGMENTS}

The authors thank Dr. I.A. Butovich, Institute for Bio-Organic and Oil Chemistry, Ukrainian Academy of Sciences (Kiev, Ukraine), for initiating the work on detergents in our group and for stimulating discussions, and Dr. S.R. Martin, National Institute for Medical Research (London, United Kingdom) for valuable suggestions concerning the interpretation of the data and for critically reading the manuscript. M.J.S. is supported by a fellowship under European Community Science Program Twinning Grant SC1-0197 to G.A.V., J.F.G.V., A. Finazzi-Agrò and L. Avigliano. 


\section{NONIONIC DETERGENTS AND LIPOXYGENASE CATALYSIS}

\section{REFERENCES}

1. Veldink, G.A., and Vliegenthart, J.F.G. (1984) in Advances in Inorganic Biochemistry (Eichhorn, G.L., and Marzili, L.G., eds.) Vol. V, pp. 139-161, Elsevier, Amsterdam.

2. Schewe, T., Rapoport, S.M., and Kühn, H. (1986) Adv. Enzymol. Relat. Areas Mol. Biol. 58, 191-272.

3. Kühn, H., Schewe, T., and Rapoport, S.M. (1986) Adv. Enzymol. Relat. Areas Mol. Biol. 58, 273-311.

4. Verhagen, J., Vliegenthart, J.F.G., and Boldingh, J. (1978) Chem. Phys. Lipids 22, 255-259.

5. Zimmerman, G.L., and Snyder, H.E. (1974) J. Agricult. Food Chem. 22, 802-805.

6. Bild, G.S., Ramadoss, C.S., and Axelrod, B. (1977) Lipids 12, $732-735$.

7. Butovich, I.A., and Kukhar', V.P. (1989) Ukr. Biokhim. Zh. 61, 106-108.

8. Srinivasulu, S., and Appu Rao, A.G. (1993) J. Agric. Food Chem. 41, 366-371.

9. Galpin, J.R., and Allen, J.C. (1977) Biochim. Biophys. Acta 488, 392-401.

10. Perez-Gilabert, M., Sanchez-Ferrer, A., and Garcia-Cramona, F. (1992) Biochem. J. 288, 1011-1015.

11. Schilstra, M.J., Veldink, G.A., Verhagen, J., and Vliegenthart, J.F.G. (1992) Biochemistry 31, 7692-7699.

12. Ludwig, P., Holzhütter, H.-G., Colosimo, A., Silvestrini, M.C., Schewe, T., and Rapoport, S.M. (1987) Eur. J. Biochem. 168, 325-337.

13. Schilstra, M.J., Veldink, G.A., and Vliegenthart, J.F.G. (1993) Biochemistry 32, 7686-7691.
14. Press, W.H., Flannery, B.P., Teukolsky, S.A., and Vetterling, W.T. (1989) in Numerical Recipes. The Art of Scientific Computing, pp. 523-528, Cambridge University Press, Cambridge.

15. Aoshima, H., Kajiwara, T., Hanataka, A., Nakatani, H., and Hiromi, K. (1977) Biochim. Biophys. Acta 486, 121-126.

16. McGhee, J.D., and Von Hippel, P.H. (1974) J. Mol. Biol. 86, 469-489.

17. Helenius, A., and Simons, K. (1975) Biochim. Biophys. Acta 415, 29-79.

18. Helenius, A., McCaslin, D.R., Fries, E., and Tanford, C. (1979) Meth. Enzymol. 56, 734-749.

19. Tanford, C. (1980) in The Hydrophobic Effect: Formation of Micelles and Biological Membranes, pp. 81-85, John Wiley and Sons, New York.

20. Christopher, J.P., Pistorius, E.K., and Axelrod, B. (1970) Biochim. Biophys. Acta 198, 12-19.

21. Van Os, C.P.A., Rijke-Schilder, G.P.M., and Vliegenthart, J.F.G. (1979) Biochim. Biophys. Acta 575, 479-484.

22. Garssen, G.J., Vliegenthart, J.F.G., and Boldingh, J. (1972) Biochem. J. 130, 435-442.

23. Butovich, I.A., and Kukhar', V.P. (1991) Doklady Biochemistryy 316, 33-37.

24. Kühn, H., Belkner, J., Wiesner, R., and Brash, A.R. (1990) J. Biol. Chem. 265, 18351-18358.

[Received October 26, 1993, and in revised form January 20, 1994; Revision accepted February 10, 1994] 